\title{
Computation of arbitrarily constrained synthetic discriminant functions
}

\author{
Mario Montes-Usategui, Juan Campos, and Ignacio Juvells
}

\begin{abstract}
An algorithm for computing correlation filters based on synthetic discriminant functions that can be displayed on current spatial light modulators is presented. The procedure is nondivergent, computationally feasible, and capable of producing multiple solutions, thus overcoming some of the pitfalls of previous methods.

Key words: Optical pattern recognition, VanderLugt correlators, synthetic discriminant functions, arbitrarily constrained filters.
\end{abstract}

\section{Introduction}

The design of filters for VanderLugt correlators has a strong mathematical foundation through the synthetic discriminant function (SDF) theory. ${ }^{1}$ The modulation of the system response provided by SDF filters is a powerful technique for solving patternrecognition problems and makes the design of optical correlation filters similar to the training procedures used for neural networks. No particular knowledge about the structure of the problem is needed, and only a representative set of examples and solutions must be obtained.

Furthermore, the theory is general enough to encompass, in particular cases, many filters obtained on an independent basis, outside the SDF philosophy; that is, many well-known filters can be obtained through the SDF theory by a proper selection of training images and desired outputs. For example, minimum average correlation energy (MACE), minimum variance SDF's, and optimum trade-off SDF's become inverse, matched, and Wiener filters, respectively, when only one image is included in the training set. $^{2-4}$ By designing an SDF filter with enough in-plane rotated images, we obtain a circular harmonic filter if the outputs are properly selected. ${ }^{5}$

M. Montes-Usategui and I. Juvells are with the Departament de Física Aplicada i Electrònica, Laboratori d'Optica, Universitat de Barcelona, Diagonal 647, E-08028 Barcelona, Spain. J. Campos is with the Departament de Física, Grup d'Optica, Universitat Autònoma de Barcelona, E-08193 Bellaterra, Spain.

Received 7 October 1994; revised manuscript received 9 January 1995.

0003-6935/95/203904-11\$06.00/0.

(1) 1995 Optical Society of America.
However, until recently, little attention has been paid to the restrictions that current technology imposes on the filter values. Modulators are essential in the construction of reliable correlators when the filter must be frequently updated and generalpurpose systems are required to do so many times per second. Unfortunately, current devices can display only a small fraction of the complex values required by the classical designs. The extreme case is that of binary modulators, in which only two values can be selected and with which most filters behave quite differently from their full-complex counterparts. In this context, Juday ${ }^{6}$ and recently Laude and Réfrégier ${ }^{7}$ have addressed the problem of the optimum projection of single-image filters to the domain allowed by a modulator.

For SDF filters with several images in the training set, the problem is worse, because a simple projection of the values of the fully complex filter will, in most cases, dramatically modify the desired outputs. Therefore the constraints imposed by the filter plane modulator must be considered in the design of the filter.

The majority of the efforts has been directed at obtaining phase-only and binary-phase-only SDF filters, although the general case of arbitrary constraints has also been studied. The first attempt to design a phase-only SDF was reported by Horner and Gianino, ${ }^{8}$ whose solution consisted simply of using the phase of a conventional composite filter. Although the first tests gave good results, the approach is not appropriate because the SDF constraints are no longer met. ${ }^{9}$ Since then a variety of formulas have appeared in the literature but none of them seem to give the ultimate answer: Kallman's algorithm ${ }^{10}$ is computationally expensive and gives 
little control over the correlation peaks (according to Ref. 11). The procedures proposed by Jared and Ennis ${ }^{11}$ and Bahri and Kumar ${ }^{12}$ limit the number of possible solutions by supposing the filter to be a linear combination of the training images, thus affecting the probability of convergence. The Jared and Ennis algorithm has also been applied to design arbitrarily constrained filters. ${ }^{13}$ The entropy-optimized filter proposed by Mahlab and Shamir ${ }^{14}$ uses the simulated annealing algorithm, inheriting its drawbacks: heuristic selection of several parameters (initial temperature, number of iterations until thermal equilibrium, etc.) and a high computational load (Ref. 5 reports $7 \mathrm{~h}$ $30 \mathrm{~min}$ on a VAX8200 computer for 10 images of $64 \times 64$ pixels).

In this paper a new algorithm for computing constrained SDF filters is proposed. It is based on a new filter design that we call a minimum Euclidean distance SDF (MED-SDF) filter, which enables us to obtain the closest SDF filter to a given non-SDF filter in the sense of Euclidean distance. This design is then used in an iterative algorithm that leads to a solution in a few steps.

The paper is organized as follows: In Section 2 we present some previous considerations about the structure of the algorithm. In particular we see the necessity of the MED-SDF filter, whose expression is deduced in Section 3. In Section 4 an sketch of the algorithm is presented as well as an analysis of the convergence properties. Section 5 shows the results of a computer simulation and finally the paper is closed by the conclusions and a mathematical appendix.

\section{Structure of the Algorithm}

We designed an iterative algorithm with a structure similar to that used in the successive forcing algorithm, ${ }^{12}$ that is, an algorithm in which, at each iteration, the filter is forced to fulfill the conditions for the central correlations and subsequently to take values on the allowed domain (Fig. 1).

This technique of successive projections is widely used as a basis of different algorithms in filter synthesis or in image restoration. ${ }^{15,16}$ The answer to (1) How to project the SDF filter $\mathbf{h}_{k}$ onto the domain allowed by the modulator to obtain filter $\mathbf{a}_{k}$ and (2) how to force the constrained filter $\mathbf{a}_{k}$ to give the desired central correlations, that is, to obtain the SDF filter $\mathbf{h}_{k+1}$, will completely shape the algorithm.

Among all the possibilities, we are interested in those that lead to convergent procedures. Because we are looking for the SDF filters that take values



Fig. 1. Structure of the algorithm. CONS, constrained filter. on the specified subset of the complex unit circle, such convergence should be expressed as

$$
\sum_{i=1}^{N}\left|h_{k+1}^{i}-a_{k+1}^{i}\right|^{2}<\sum_{i=1}^{N}\left|h_{k}^{i}-a_{k}^{i}\right|^{2},
$$

where $\mathbf{h}$ and $\mathbf{a}$ are the SDF and the constrained filters, respectively. The subscripts $k$ and $k+1$ indicate the iteration, and the superscript $i$ indicates the pixel; finally, $N$ represents the number of pixels of $\mathbf{h}$ and a. Inequality (1) demands that some measure of similarity between the SDF and the constrained filter be a quantity decreasing with the number of iterations. Although it has been written with the Euclidean distance as a measure of similarity, this is not the only possibility and other metrics might be of use. The solution will be reached when the distance between the two filters drop under some limit, depending on the desired precision for the correlation values.

However, the imposition of inequality (1) implies that one solution always exists and that such a solution can be reached by means of an algorithm with the chosen structure. We are not able to ensure this so we had to use the less ambitious condition

$$
\sum_{i=1}^{N}\left|h_{k+1}^{i}-a_{k+1}^{i}\right|^{2} \leq \sum_{i=1}^{N}\left|h_{k}^{i}-a_{k}^{i}\right|^{2}
$$

which is similar to inequality (1) but in which the equality sometimes may hold.

By splitting expression (2) into

$$
\sum_{i=1}^{N}\left|h_{k+1}^{i}-a_{k+1}^{i}\right|^{2} \leq \sum_{i=1}^{N}\left|h_{k+1}^{i}-a_{k}^{i}\right|^{2} \leq \sum_{i=1}^{N}\left|h_{k}^{i}-a_{k}^{i}\right|^{2}
$$

in which expression (2) is obviously implied, we can answer the two questions stated above in an unique way.

The first inequality in expression (3),

$$
\sum_{i=1}^{N}\left|h_{k+1}^{i}-a_{k+1}^{i}\right|^{2} \leq \sum_{i=1}^{N}\left|h_{k+1}^{i}-a_{k}^{i}\right|^{2},
$$

is used to project the SDF filter onto the allowed domain of the complex plane. At this point of the process we know everything except the filter $\mathbf{a}_{k+1}$. Expression (4) compares the distance of $\mathbf{a}_{k}$ and $\mathbf{a}_{k+1}$ with respect to the SDF filter at iteration $k+1, \mathbf{h}_{k+1}$. Because $\mathbf{a}_{k}$ is a constrained filter, if we choose $\mathbf{a}_{k+1}$ as the constrained filter, that makes minimum

$$
E(\mathbf{a})=\sum_{i=1}^{N}\left|h_{k+1}^{i}-a^{i}\right|^{2}
$$

expression (4) is automatically satisfied. Equation (5) is a sum of positive and independent terms and 
therefore the minimum is reached by the minimization of each addend. As the coding domain is assumed to be known, the process merely reduces to a search of the closest domain value to each component of $\mathbf{h}_{k+1}$ (Fig. 2). For example, for a phase-only filter, $\mathbf{a}_{k+1}$ is obtained by the extraction of the phase of the SDF filter $\mathbf{h}_{k+1}$. It is worth pointing out that this process is used to project, in an optimum way, a single-image filter. ${ }^{6,7}$

Equivalently, the second expression,

$$
\sum_{i=1}^{N}\left|h_{k+1}^{i}-a_{k}^{i}\right|^{2} \leq \sum_{i=1}^{N}\left|h_{k}^{i}-a_{k}^{i}\right|^{2},
$$

tells us how to derive the SDF filter at the next iteration, $\mathbf{h}_{k+1}$, from the constrained filter at the previous iteration, $\mathbf{a}_{k}$. At this point we know everything except $\mathbf{h}_{k+1}$. Expression (6) compares the distance of $\mathbf{h}_{k}$ and $\mathbf{h}_{k+1}$ with respect to the constrained filter at iteration $k, \mathbf{a}_{k}$. Because $\mathbf{h}_{k}$ is an SDF filter, when $\mathbf{h}_{k+1}$ is selected as the SDF filter, which makes

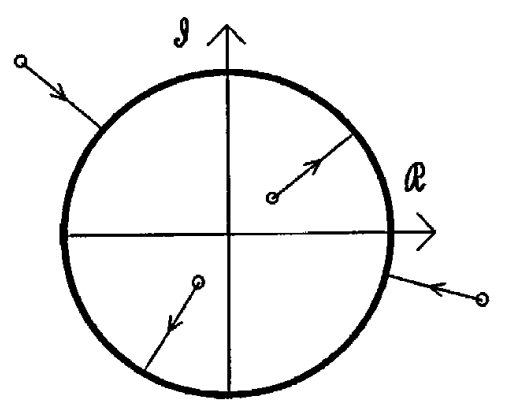

(a)

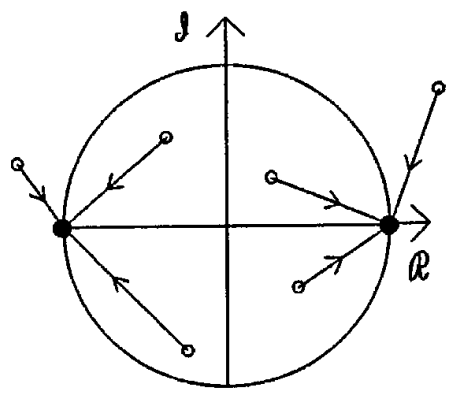

(b)

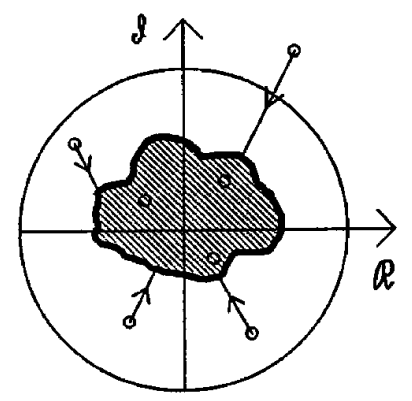

(c)

Fig. 2. Projection of the filter values onto the allowed domain for (a) a phase-only filter, (b) a binary-phase-only filter, (c) an arbitrary domain. minimum

$$
E(\mathbf{h})=\sum_{i=1}^{N}\left|h^{i}-a_{k}^{i}\right|^{2},
$$

the inequality in expression $(6)$ is automatically met. The process for obtaining such a filter is not as evident as before because now the terms of the sum are not independent; they are linked together by the conditions imposed on the correlations with the training images. The appropriate conditions and the resulting expression for the MED-SDF filter is given in Section 3.

Figure 3 summarizes the information contained in this section. Starting from the SDF filter at iteration $k, \mathbf{h}_{k}$, we obtain the constrained filter $\mathbf{a}_{k}$ by looking, among all possible constrained filters, for the closest to $\mathbf{h}_{k}$. We force this filter to be an SDF design (to obtain $\mathbf{h}_{k+1}$ ) by looking, among all possible SDF filters, for the closest to $\mathbf{a}_{k}$. Note that $\mathbf{h}_{k+1}$ is not necessarily equal to the original filter $\mathbf{h}_{k}$ and, when they coincide, the algorithm stops. This issue is discussed in Section 4. The latter step is carried out by the computation of the MED-SDF filter.

\section{Minimum Euclidean Distance Synthetic Discriminant Function}

Let us suppose we have a correlation filter a that we wish to modify to give some prespecified values for the central correlations with $M$ images. The shape of the filter is important, and so we want to change it as little as possible. The question as to which filter enables us to obtain the desired correlations by preserving the original filter a as far as possible is answered in this section. We call this design the minimum Euclidean distance synthetic discriminant function (MED-SDF). The problem can be stated in the following terms:

Let $\mathbf{X}_{1}, \ldots, \mathbf{X}_{M}$ denote the Fourier transforms of the $M$ images of $N$ components for which we wish to obtain the values $c_{1}, \ldots, c_{M}$ at the center of the correlation plane. Let $\mathbf{a}$ be the filter we need to

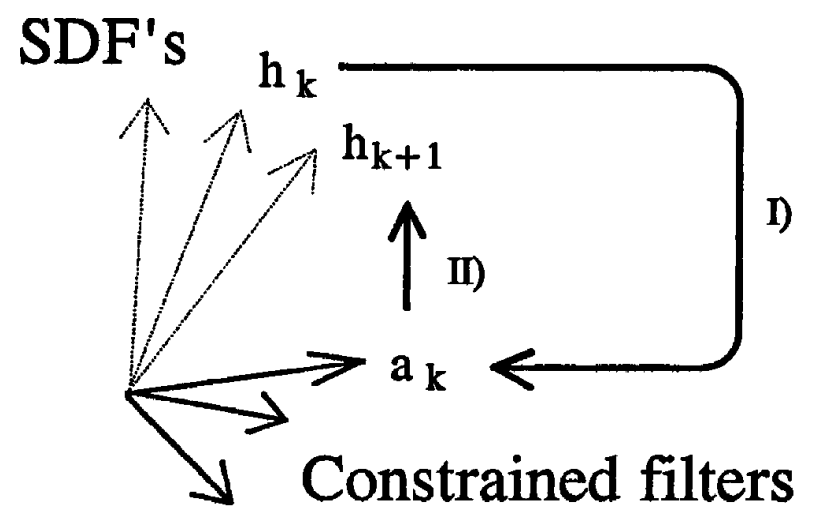

Fig. 3. Sketch of the two successive projections that form an iteration of the algorithm. 
modify. We are looking for the filter $\mathbf{h}$ so that

$$
\mathbf{h}^{+} \mathbf{X}=\mathbf{c}^{T},
$$

where

$$
E(\mathbf{h})=\sum_{i=1}^{N}\left|h^{i}-a^{i}\right|^{2}
$$

is a minimum.

In Eq. (8), $\mathbf{X}$ is the $N \times M$ matrix whose columns are the images $\mathbf{X}_{i}, \mathbf{c}$ is the column vector of $M$ components containing the values $c_{i}$, and the superscripts $T$ and + mean transpose and conjugate transpose, respectively. Finally, $h^{i}$ and $a^{i}$ represent the component number $i$ of $\mathbf{h}$ and $\mathbf{a}$, respectively. Equation (9) can be rewritten with vector notation as

$$
\begin{aligned}
\mathbf{E}(\mathbf{h})= & \sum_{i=1}^{N}\left[\left(h^{i}-a^{i}\right) *\left(h^{i}-a^{i}\right)\right] \\
= & \sum_{i=1}^{N}\left[\left(h^{i}\right)^{*} h^{i}-\left(h^{i}\right)^{*} a^{i}\right. \\
& \left.-\left(a^{i}\right)^{*} h^{i}+\left(a^{i}\right)^{*} a^{i}\right] \\
= & \mathbf{h}^{+} \mathbf{h}-\mathbf{h}^{+} \mathbf{a}-\mathbf{a}^{+} \mathbf{h}+\mathbf{a}^{+} \mathbf{a} .
\end{aligned}
$$

If the real and the imaginary parts of all the quantities are made explicit, namely $\mathbf{h}=\mathbf{h}_{R}+j \mathbf{h}_{I}$, $\mathbf{a}=\mathbf{a}_{R}+j \mathbf{a}_{I}, \mathbf{X}=\mathbf{X}_{R}+j \mathbf{X}_{I}$, and $\mathbf{c}=\mathbf{c}_{R}+j \mathbf{c}_{I}$, where $j$ is the imaginary unit, Eqs. (8) and (10) become

$$
\begin{aligned}
\mathbf{h}_{R}^{T} \mathbf{X}_{R}+\mathbf{h}_{I}^{T} \mathbf{X}_{I}= & \mathbf{c}_{R}^{T}, \\
\mathbf{h}_{R}^{T} \mathbf{X}_{I}-\mathbf{h}_{I}^{T} \mathbf{X}_{R}= & \mathbf{c}_{I}^{T}, \\
E\left(\mathbf{h}_{R}, \mathbf{h}_{I}\right)= & \mathbf{h}_{R}^{T} \mathbf{h}_{R}+\mathbf{h}_{I}^{T} \mathbf{h}_{I}-2 \mathbf{h}_{R}^{T} \mathbf{a}_{R} \\
& -2 \mathbf{h}_{I}^{T} \mathbf{a}_{I}+\mathbf{a}_{R}^{T} \mathbf{a}_{R}+\mathbf{a}_{I}^{T} \mathbf{a}_{I} .
\end{aligned}
$$

We can find the solution by setting the gradients of the Lagrange function $L\left(\mathbf{h}_{R}, \mathbf{h}_{I}\right)$ to 0 with respect to the filter components, where,

$$
\begin{aligned}
L\left(\mathbf{h}_{R}, \mathbf{h}_{I}\right)= & \mathbf{h}_{R}^{T} \mathbf{h}_{R}+\mathbf{h}_{I}^{T} \mathbf{h}_{I}-2 \mathbf{h}_{R}^{T} a_{R} \\
& -2 \mathbf{h}_{I}^{T} a_{I}+\mathbf{a}_{R}^{T} \mathbf{a}_{R}+\mathbf{a}_{I}^{T} \mathbf{a}_{I} \\
& -2\left(\mathbf{h}_{R}^{T} \mathbf{X}_{R}+\mathbf{h}_{I}^{T} \mathbf{X}_{I}-\mathbf{c}_{R}^{T}\right) \mathbf{u} \\
& -2\left(\mathbf{h}_{R}^{T} \mathbf{X}_{I}-\mathbf{h}_{I}^{T} \mathbf{X}_{R}-\mathbf{c}_{I}^{T}\right) \mathbf{v}
\end{aligned}
$$

and $\mathbf{u}$ and $\mathbf{v}$ are $M$-dimensional column vectors containing the Lagrange multipliers. Such a solution can be written in vector notation as

$$
\mathbf{h}=\mathbf{a}+\mathbf{X w}^{*},
$$

with $\mathbf{w}=\mathbf{u}+j \mathbf{v}$. Substitution of Eq. (14) into Eq. (8) leads to

$$
\left(\mathbf{a}^{+}+\mathbf{w}^{T} \mathbf{X}^{+}\right) \mathbf{X}=\mathbf{c}^{T},
$$

whence

$$
\mathbf{w}^{*}=\left(\mathbf{X}^{+} \mathbf{X}\right)^{-1}\left(\mathbf{c}^{*}-\mathbf{X}^{+} \mathbf{a}\right) .
$$

Substituting Eq. (16) into Eq. (14), we finally obtain

$$
\begin{aligned}
\mathbf{h} & =\mathbf{X}\left(\mathbf{X}^{+} \mathbf{X}\right)^{-1} \mathbf{c}^{*}+\left[\mathbf{I}_{N}-\mathbf{X}\left(\mathbf{X}^{+} \mathbf{X}\right)^{-1} \mathbf{X}^{+}\right] \mathbf{a} \\
& \equiv \mathbf{c o m p}+\mathbf{P a},
\end{aligned}
$$

where $\mathbf{I}_{N}$ is the $N \times N$ identity matrix. Equation $(17)$ can be rewritten as

$$
\mathbf{h}=\mathbf{a}+\Delta \mathbf{a}=\mathbf{a}+\mathbf{X}\left(\mathbf{X}^{+} \mathbf{X}\right)^{-1}\left(\mathbf{c}^{*}-\mathbf{X}^{+} \mathbf{a}\right),
$$

that is, the modification of filter $\mathbf{a}$ is a composite filter that complements the central correlations in the exact amount needed. Because the composite filter is, among the SDF's, that with minimum modulus (it minimizes $\mathbf{h}^{+} \mathbf{h}$ ), it changes the original filter $\mathbf{a}$ as little as possible. The expression in Eq. (17) admits a potentially useful interpretation. When $\mathbf{a}$ is an arbitrarily chosen vector, Eq. (17) represents the most general solution to the SDF problem, ${ }^{17}$ where the term $\mathbf{X}\left(\mathbf{X}^{+} \mathbf{X}\right)^{-1} \mathbf{c}^{*}$ is the classical composite filter and $\mathbf{I}_{N}-\mathbf{X}\left(\mathbf{X}^{+} \mathbf{X}\right)^{-1} \mathbf{X}{ }^{+}$is the projection operator over the subspace spanned by the $N-M$ orthogonal vectors to the training images. Every SDF design can be expressed in the above form by the proper choice of the vector $\mathbf{a}$, which can now be interpreted as the filter to which $\mathbf{h}$ most approximates, thus establishing an interesting link between SDF and non-SDF filters.

\section{Algorithm}

The whole process to compute constrained SDF filters is depicted in Fig. 4 and can be sketched as follows:

Step 1: Choose an initial vector $\mathbf{a}_{0}$ of $N$ components.

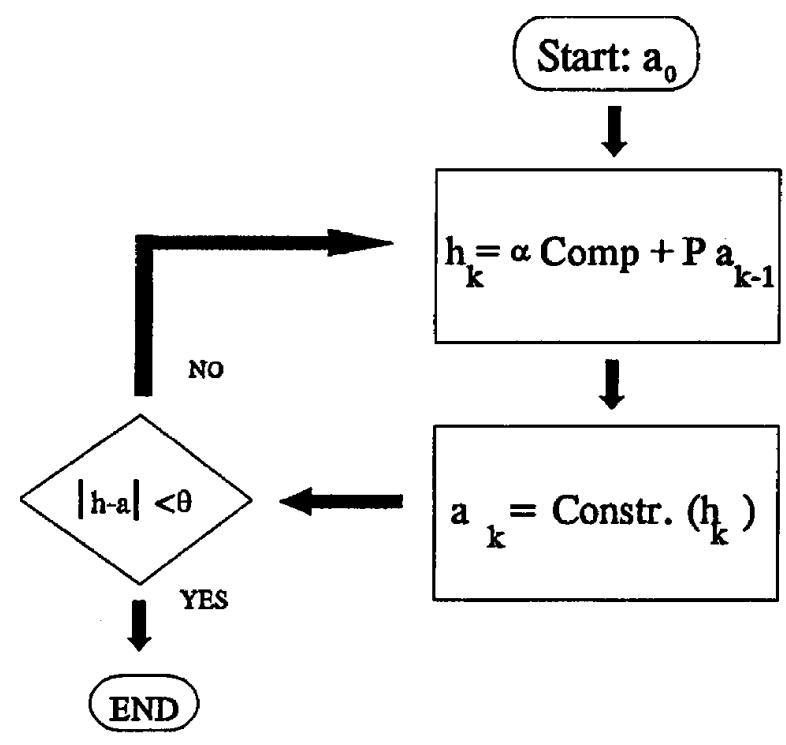

Fig. 4. Block diagram of the proposed algorithm.

10 July 1995 / Vol. 34, No. 20 / APPLIED OPTICS 
Step 2: Start iteration $k:$ compute

$$
h_{k}=\alpha \operatorname{comp}+\mathbf{P a}_{k-1}
$$

by means of Eq. (17), where $\alpha$ is a scaling constant that is justified below.

Step 3: Compute for $i=1$ to $N$ :

$$
a_{k}^{i}=\arg \min _{\forall s \in D}\left(\left|h_{k}^{i}-s\right|^{2}\right)
$$

where $D$ represents the coding domain and $s$ is an arbitrary value of this domain.

Step 4: If the difference between the constrained and the SDF filters is small or if the algorithm stops its convergence, i.e., if

$$
E_{k}=\sum_{i=1}^{N}\left|h_{k}^{i}-a_{k}^{i}\right|^{2}<\theta_{f}
$$

or

$$
\left|E_{k}-E_{k-1}\right|<\theta_{m}
$$

then exit; $\theta_{f}$ and $\theta_{m}$ are arbitrarily chosen small numbers.

Step 5: If the condition in step 4 is not satisfied, finish iteration $k$ by going to step 2 .

The computation of the MED-SDF filter is carried out in step 2. It involves a matrix-vector multiplication to project the constrained filter onto the orthogonal subspace to the training images and the addition of the resulting vector to the composite filter. Note that both this filter and the projection matrix are fixed and can be precomputed and stored. However, the projection operator is a matrix of $N \times N$ components and would require a huge amount of memory (for $128 \times 128$ images it needs over 1 Gbyte). A good compromise between memory requirements and computation complexity is to precalculate only $S=$ $\mathbf{X}\left(\mathbf{X}^{+} \mathbf{X}\right)^{-1}$, which is an $N \times M$ matrix (for 20 images of $128 \times 128$ pixels it needs $\sim 1$ Mbyte). Then the computation is completed at each iteration when the stored matrix is multiplied by the $M$-dimensional vector $\mathbf{X}^{+} \mathbf{a}$ and the result is added to the $N$ dimensional vector $\mathbf{a}$ :

$$
\begin{aligned}
{\left[\mathbf{I}-\mathbf{X}\left(\mathbf{X}^{+} \mathbf{X}\right)^{-1} \mathbf{X}^{+}\right] \mathbf{a} } & =\mathbf{a}-\mathbf{X}\left(\mathbf{X}^{+} \mathbf{X}\right)^{-1} \mathbf{X}^{+} \mathbf{a} \\
& =\mathbf{a}-\mathbf{S}\left(\mathbf{X}^{+} \mathbf{a}\right) .
\end{aligned}
$$

The scaling constant in step 2 provides an additional degree of freedom. It accounts for the fact that the specified values for the central correlations can be rescaled to obtain a better match between the computed filter and the values available in the coding domain. We want a bright correlation spot with the target images and a dim one with the nontarget patterns, but the exact value to be imposed at the center of the correlation plane to achieve this depends a good deal on the characteristics of the filter plane modulator. In appendix A we deduce the expression for the scaling constant that leads to a minimum distance between $\mathbf{h}_{k+1}$ and $\mathbf{a}_{k}$. Note that we have two additional sources of degrees of freedom:

- The phases of the central correlations with the $M$ training images can be used to minimize further the difference between the SDF filter $\mathbf{h}_{k+1}$ and the constrained filter $\mathbf{a}_{k}$. The same procedure proposed for optimizing the phase in previous SDF designs can be used. ${ }^{18,19}$

- The SDF filter $\mathbf{h}_{k+1}$ can be rescaled again by a complex constant to obtain $\mathbf{a}_{k+1}$ with minimal error, as proposed in Ref. 6 for single-image filters.

We did not exploit these two possibilities in order to not overly complicate the algorithm. Although the nondivergent behavior of the algorithm will not be affected by not performing these additional operations the probability of convergence is lower.

The process stops only when (a) one solution is reached; the constrained filter $\mathbf{a}_{k}$ fulfills the SDF conditions, i.e.,

$$
\mathbf{a}_{k}=\mathbf{c o m p}+\mathbf{P v},
$$

where $\mathbf{v}$ is some $N$-dimensional vector. Filter $\mathbf{h}_{k+1}$ is then

$$
\begin{aligned}
\mathbf{h}_{k+1} & =\operatorname{comp}+\mathbf{P}(\operatorname{comp}+\mathbf{P v}) \\
& =\operatorname{comp}+\mathbf{P}(\mathbf{c o m p})+\mathbf{P}^{2} \mathbf{v} \\
& =\operatorname{comp}+\mathbf{0}+\mathbf{P v}=\mathbf{a}_{k},
\end{aligned}
$$

and the output is constant from this point on. We derived Eq. (25) by using $\mathbf{P}$ as a projection operator, and therefore $\mathbf{P}^{2}=\mathbf{P}$, and the filter comp as a linear combination of the training images and thus its projection $\mathbf{P}(\mathbf{c o m p})$ is null.

(b) If $\mathbf{a}_{k}$ is not an SDF filter but can be written as

$$
\mathbf{a}_{k}=\mathbf{a}_{k-1}+\mathbf{v}
$$

where $\mathbf{v}$ belongs to the kernel of the projection matrix, i.e., $\mathbf{v} \in \operatorname{ker}(\mathbf{P})$. Filter $\mathbf{h}_{k+1}$ is then

$$
\begin{aligned}
\mathbf{h}_{k+1} & =\operatorname{comp}+\mathbf{P} \mathbf{a}_{k}=\mathbf{c o m p}+\mathbf{P}\left(\mathbf{a}_{k-1}+\mathbf{v}\right) \\
& =\operatorname{comp}+\mathbf{P} \mathbf{a}_{k-1}=\mathbf{h}_{k},
\end{aligned}
$$

whence

$$
\begin{aligned}
\mathbf{a}_{k} & =\mathbf{a}_{k+1}=\mathbf{a}_{k+2}=\ldots, \\
\mathbf{h}_{k} & =\mathbf{h}_{k+1}=\mathbf{h}_{k+2}=\ldots,
\end{aligned}
$$

and the distance between the SDF and the constrained filter remains constant. This possibility is unlikely, as the dimension of the kernel of the projection matrix is $M$, the number of training images, which is in general much smaller than the dimension of the space, the bandwidth product $N$.

(c) If $\mathbf{a}_{k}$ is not an SDF filter but

$$
\begin{aligned}
\mathbf{h}_{k} & \neq \mathbf{h}_{k+1}, \\
\mathbf{a}_{k} & =\mathbf{a}_{k+1},
\end{aligned}
$$


then

$$
\begin{aligned}
\mathbf{a}_{k} & =\mathbf{a}_{k+1}=\mathbf{a}_{k+2}=\ldots, \\
\mathbf{h}_{k+1} & =\mathbf{h}_{k+2}=\ldots,
\end{aligned}
$$

i.e., when the projection of two consecutive and different SDF filters is the same, the algorithm stops its convergence. This possibility is difficult to analyze and depends on the coding domain. It represents the intuitive notion that the smaller the number of coding values the smaller the probability of finding a solution. When there are no restrictions and the entire complex plane is available, the SDF and the constrained filters are always equal and expression 29 is self-contradictory and never holds. When only one coding value is allowed, all the constrained filters are the same and the process stops at the first iteration. No solution is possible. Binary modulators permit the coding of $2^{N}$ different filters, which for $128 \times 128$ images is greater than $10^{4900}$. In spite of this seemingly large number, because two consecutive SDF filters may be similar, especially when we are near the solution, their binarization may be equal with relative ease. Although we found this problem with binary-phase-only filters we show in Section 5 that the algorithm can still produce usable filters.

\section{Results}

The algorithm was tested by means of computer simulation of the optical correlation process. Toward this end, we designed several filters to solve a two-class problem involving different views of out-ofplane rotated objects. The true class was formed by 20 images of a tank captured every $18^{\circ}$. The false class contained 20 images of a truck obtained under the same conditions. All the images were $128 \times 128$

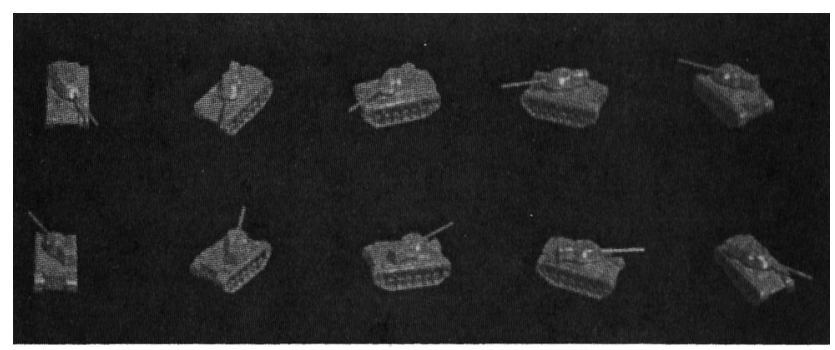

(a)

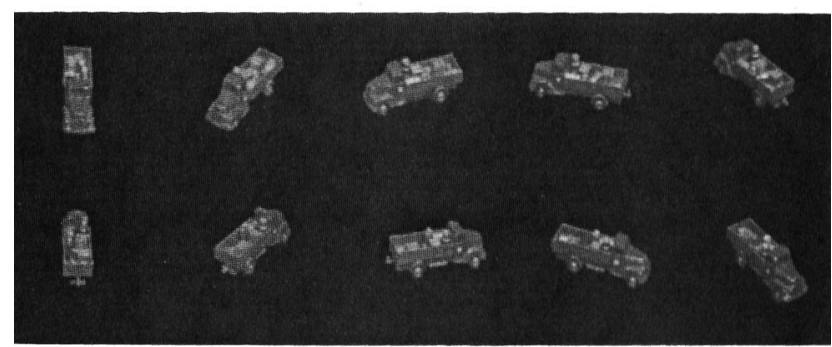

(b)

Fig. 5. Training set: (a) true-class images, (b) false-class images. pixels, and no special preprocessing such as edge enhancement was carried out. The training set, that is, the set of images used in designing the filters, for all the examples that follow is shown in Fig. 5. It is composed of the ten samples of each object taken at $0^{\circ}$, $36^{\circ}, \ldots, 324^{\circ}$ angles. The performance of the algorithm was studied for four different coding domains (Fig. 6): a phase-only domain, a binary-phase-only domain, a spiral coupling between amplitude and phase, and an arbitrary domain. Phase-only filters are attractive designs because they provide a good trade-off between noise resistance and peak sharpness together with optimum light efficiency., ${ }^{5,20}$ Binary-phase-only filters retain to a large extent the properties of the latter design, but they can be implemented in actual devices such as magneto-optic spatial light modulators, which, in addition, are very fast. The spiral domain is typical for liquid-crystal displays. Finally, Fig. 6(d) shows a rather arbitrary modulator characteristic for which the algorithm will work.

The first issue addressed was the choice of the initial filter $\mathbf{a}_{0}$. The algorithm was found to be capable of producing SDF filters with a wide variety of starting points. In particular we tested

(a) Full complex SDF filters designed to solve the same problem. We used them because sometimes the simple projection of an SDF filter is a good solution. For example, the phase of a composite filter is sometimes a good phase-only $\mathrm{SDF}^{8}$ and thus would require only small modifications.

(b) Random complex vectors. In contrast to case (a), they contain no information about the problem.

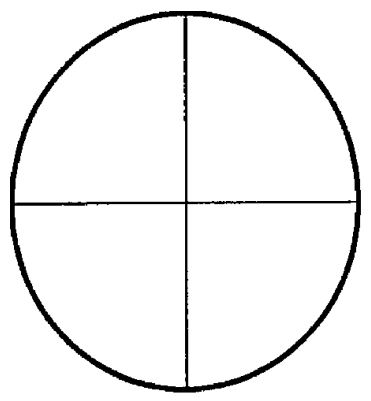

(a)

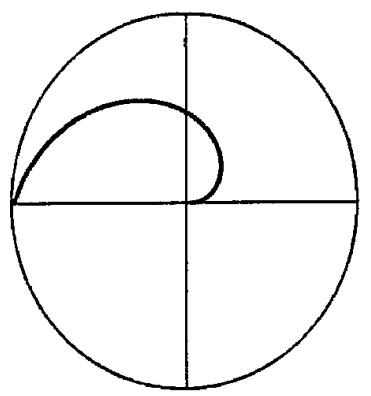

(c)

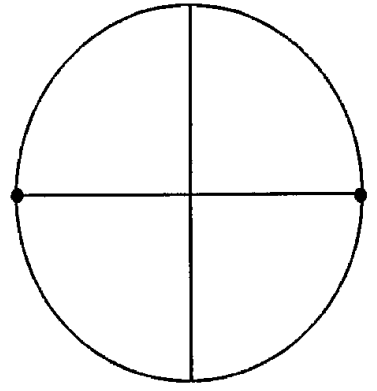

(b)

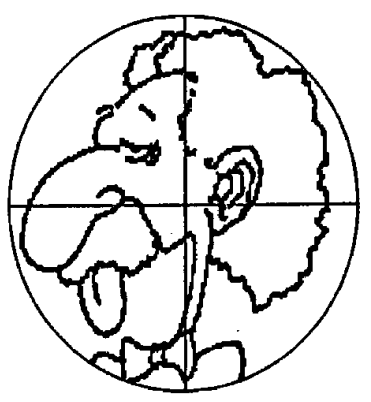

(d)
Fig. 6. Different coding domains used to test the method: (a) phase-only domain, (b) binary-phase-only domain, (c) spiral coupling between amplitude and phase, (d) arbitrary domain. 
(c) The same starting point used in the Jared and Ennis algorithm, ${ }^{11}$ i.e.,

$$
\mathbf{a}_{0}=\sum_{i=1}^{M} c_{i} \mathbf{X}_{i}
$$

where $\mathbf{X}_{i}$ is the $N$-dimensional vector representing the $i$ training image, $c_{i}$ is its desired output, and $M$ is the number of training images.

Figure 7 shows the convergence of the algorithm when a phase-only SDF is designed with a MACE filter as a starting point. The $Y$ axis, which has a logarithmic scale, represents the error between the SDF and the constrained filter at a given iteration divided by the sum of the squared magnitude of the components of the SDF filter, i.e.,

$$
E_{\text {NORM }}(\mathbf{h})=\left(\sum_{i=1}^{N}\left|h_{k}^{i}-a_{k}^{i}\right|^{2} \mid\right)\left(\sum_{i=1}^{N}\left|h_{k}^{i}\right|^{2}\right),
$$

where $\mathbf{h}$ is the SDF filter and $\mathbf{a}$ is the phase-only version of $\mathbf{h}$. The superscript $i$ indicates the component, and the subscript $k$ indicates the iteration. For an ideal phase-only filter this error function is 0 . The graph shows an exponential decay with the number of iterations, indicating a fast approach to the desired phase-only filter.

The accuracy attained for the SDF conditions was found to be only slightly dependent on the initial point, although different number of iterations (from 10 to 20) were required for different points. However, depending on $\mathbf{a}_{0}$, the behavior of the final filters may be different because the algorithm seems to find a solution easily, without modifying the starting

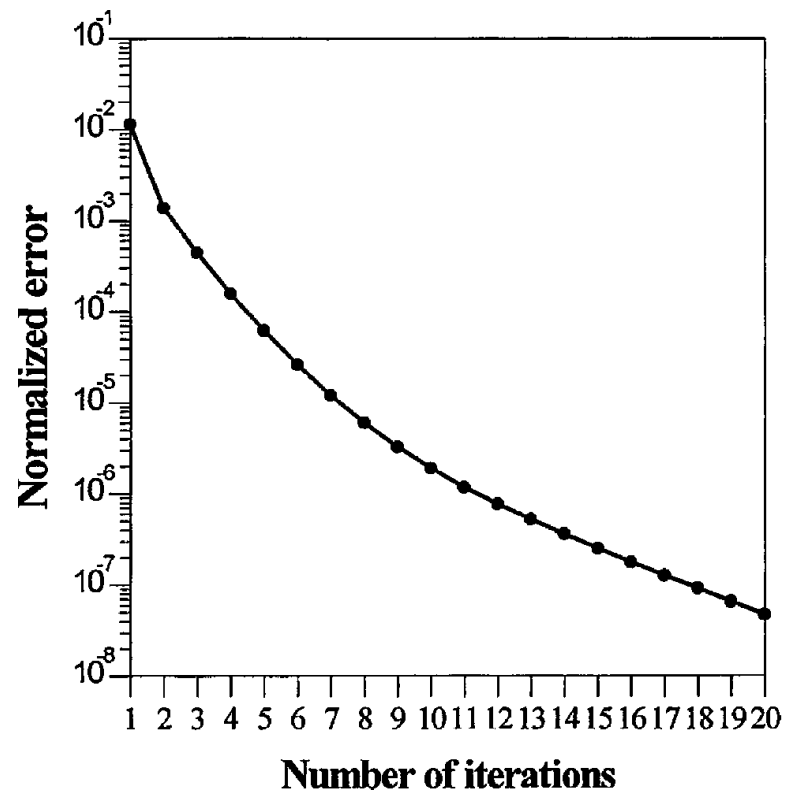

Fig. 7. Plot showing the convergence of the algorithm for a phase-only SDF. point so much. Because the solution is close to the initial filter, it preserves its characteristics to some extent.

We give two examples of this feature. In the first one, three phase-only SDF's were computed by the use of the phase of a MACE filter, a phase-only random vector, and a constant plane in Fourier space (a delta function in object space) as initial points. Figure 8 shows the impulse responses of the obtained filters. All three meet the SDF conditions with an accuracy of $96 \%$ and are very similar to their respective starting filters. For instance, the filter of Fig. $8(\mathrm{~b})$ is clearly random or the filter of Fig. 8(c) is almost a delta function. The rest of the correlation plane is, of course, very different, and this property enables a indirect control over the characteristics of the final

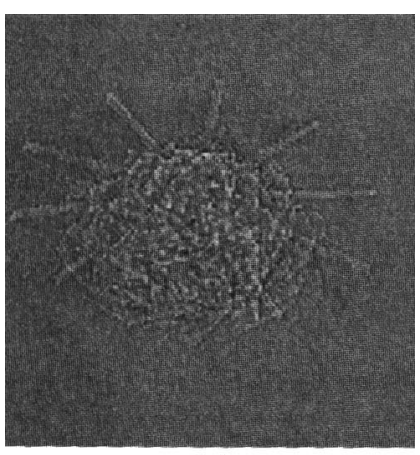

(a)

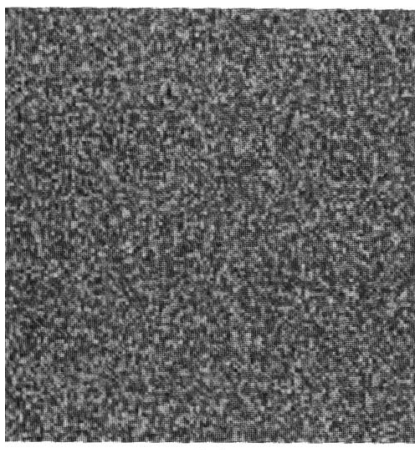

(b)

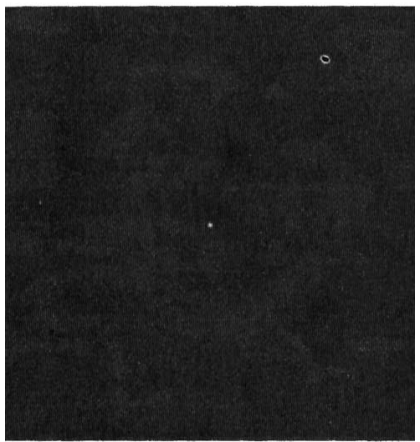

(c)

Fig. 8. Impulse response of three phase-only SDF's designed with the same training set but with different starting points: (a) the phase of a MACE filter, (b) a phase-only random vector, (c) a constant plane in Fourier space. 
filters. The second example illustrates this point more clearly.

Figure $9(\mathrm{a})$ shows the central correlations between the whole set of 40 images $(20$ tanks and 20 trucks) and a phase-only SDF designed with only the 20 views shown in Fig. 5. The starting point was the sum of the 10 target images (the 10 tanks). Note that although there is a perfect control over the central correlations with the training images and small sidelobes (see Fig. 14 below), the correlations with the

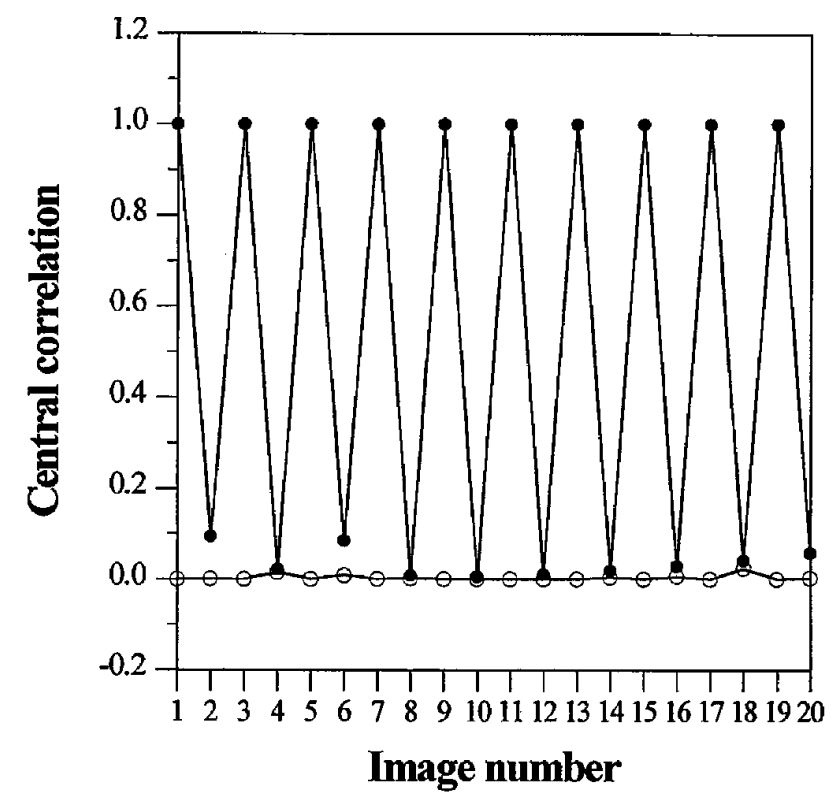

(a)

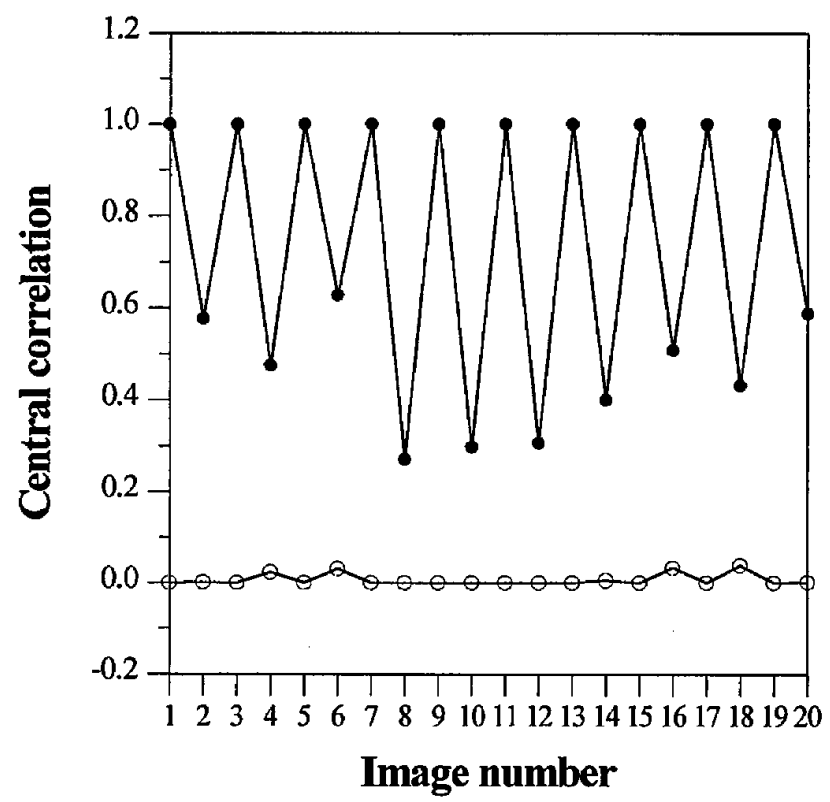

(b)

Fig. 9. Central correlations obtained with two different phaseonly SDF's: (a) with a starting point formed by the sum of the 10 tanks of the training set [Fig. 5(a)], (b) with a starting point formed by the sum of the whole set of 20 tanks. Open circles represent the correlations with the trucks. Filled circles represent those of the tanks. tanks not included in the training set are too small to be separated from those of the trucks. The true-class images can be separated from those belonging to the false class, if the initial point is formed by the sum of the whole set of true-class images ( 20 tanks), as shown in Fig. 9(b). Thus the election of an initial vector $\mathbf{a}_{0}$ that includes information about the intermediate views leads to a filter with enhanced generalization capabilities.

The type of coding domain is the most influential factor with respect to the control of the central correlations. Figures 10, 11, and 12 show respectively the central correlations between the images of the training set and the binary-phase-only filter, the spiral filter, and the arbitrarily constrained filter whose domains are shown in Fig. 6 . The correlations with the nontraining images (the intermediate views) are not shown because they strongly depend on the filter $\mathbf{a}_{0}$ as stated above. The two latter designs accurately meet the SDF constraints. The binaryphase-only filter presents more difficulties because, although the values for the true-class images are significantly higher than those of the false class, they show the most marked variation. This is due to the stop of the algorithm at approximately four iterations with all the initial points we used. Finally, Figs. 14-17 present three-dimensional plots as well as a front and a lateral view of the intensity of the correlation between the test scene of Fig. 13 and the four filters. A good detection of the tank is possible in all cases.

\section{Final Remarks and Conclusion}

A new algorithm for computing SDF's adapted to the restrictive modulation characteristics of present-day

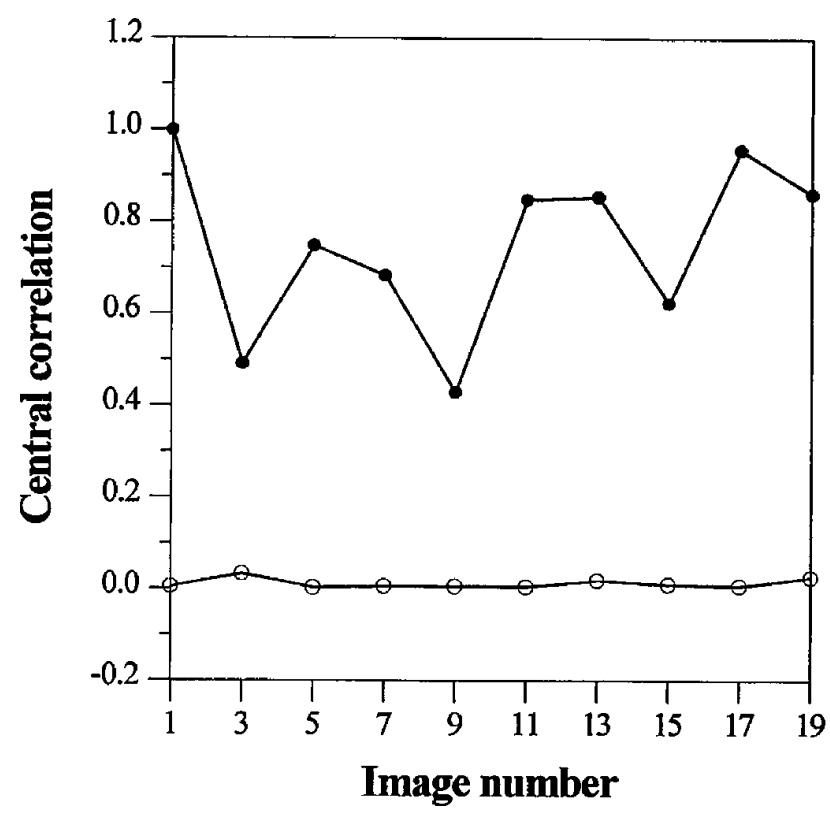

Fig. 10. Central correlations between a binary phase-only SDF and the images of the training set. Open circles represent the correlations with the trucks; filled circles represent those of the tanks. 


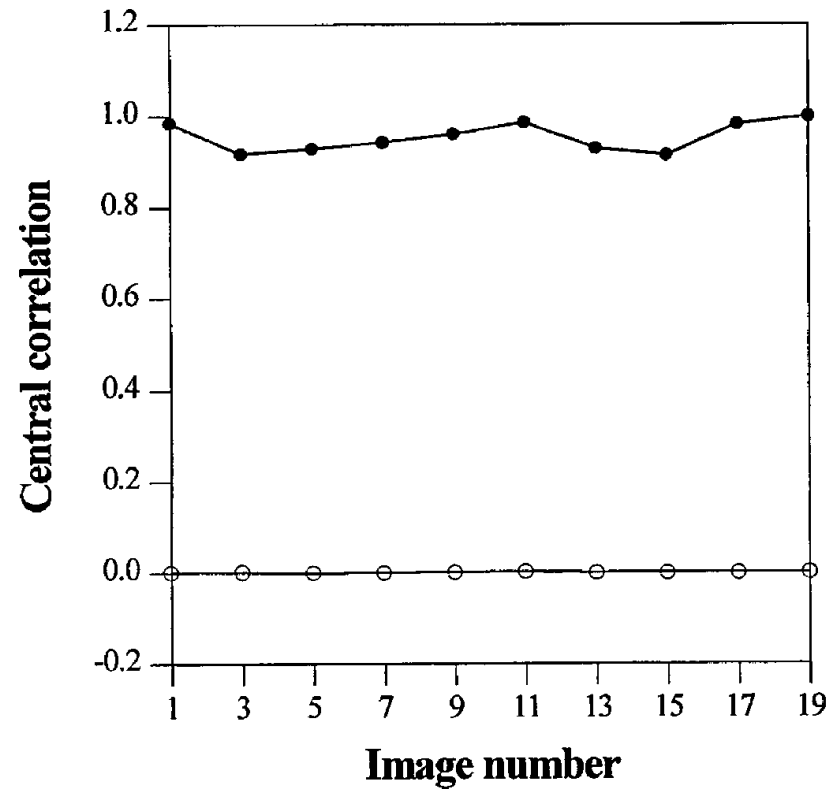

Fig. 11. Central correlations between a spirally constrained SDF filter and the images of the training set. Open circles represent the correlations with the trucks; filled circles represent those of the tanks.

devices has been developed. In contrast to other previously proposed methods, our procedure can be proved to be nondivergent. Furthermore it has a solid mathematical background that enables the analysis of the cases that do not lead to a solution. The algorithm needs only a few iterations, ranging from 10 to 20 , to obtain the desired filter so the computational load is moderate. Finally, no special assump-

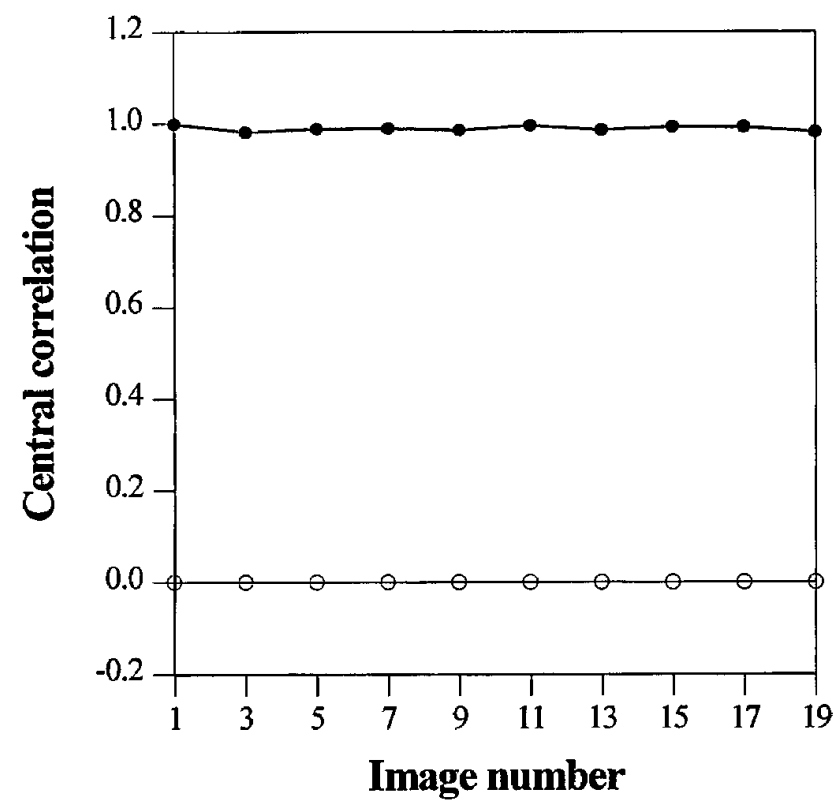

Fig. 12. Central correlations between an SDF with values on the domain of Fig. 6(d) and the images of the training set. Open circles represent the correlations with the trucks; filled circles represent those of the tanks.

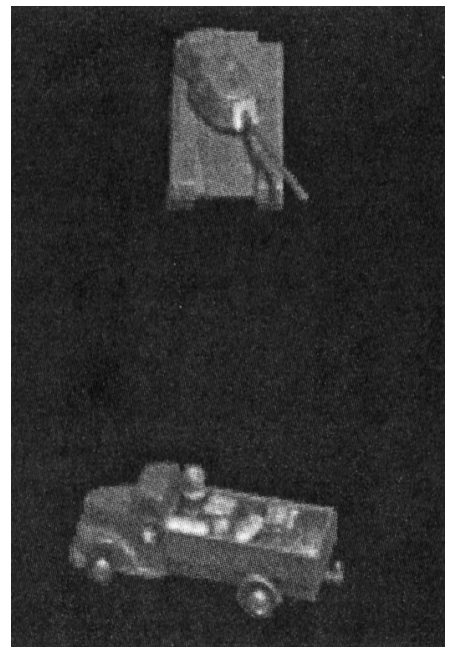

Fig. 13. Test input scene. Both images belong to the training set.

tion of the shape of the filter was made, such as the imposition for the filter to be a linear combination of the training images and therefore multiple solutions could be reached by changing the initial point. This property enables an indirect control over the characteristics of the final filter, as indicated by the results of the simulation. Nevertheless, a systematic approach for the selection of the initial filter to take full advantage of this feature must still be devised. We are currently working in this direction to introduce optimality considerations into the algorithm.

There are other possibilities worth exploring, such as the use of different metrics to measure the similar-

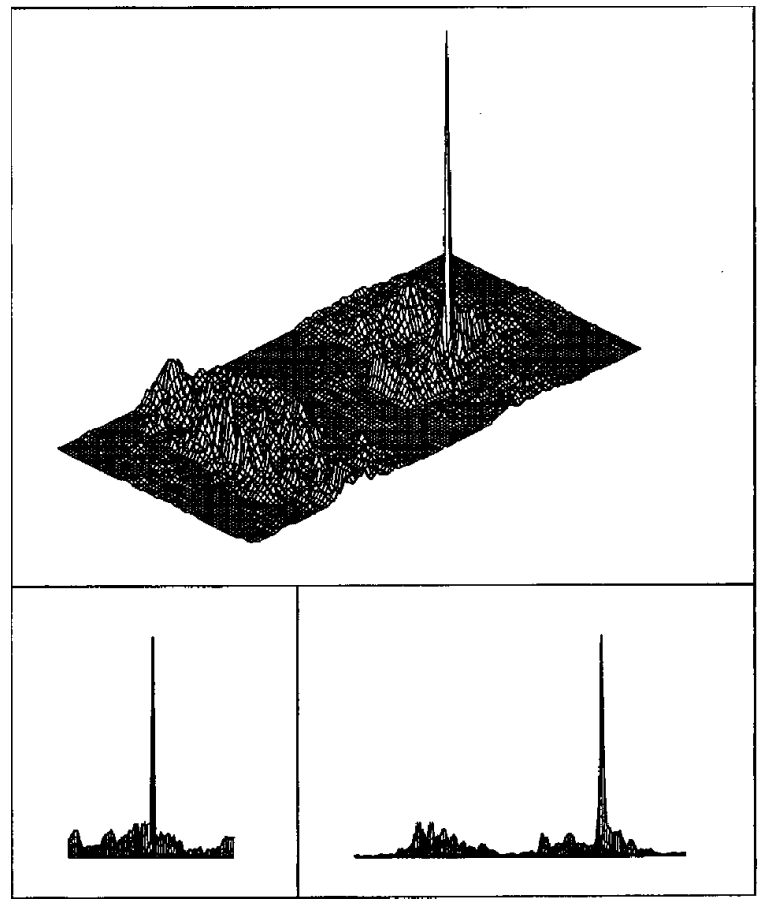

Fig. 14. Correlation between the phase-only SDF and the input scene of Fig. 13. 


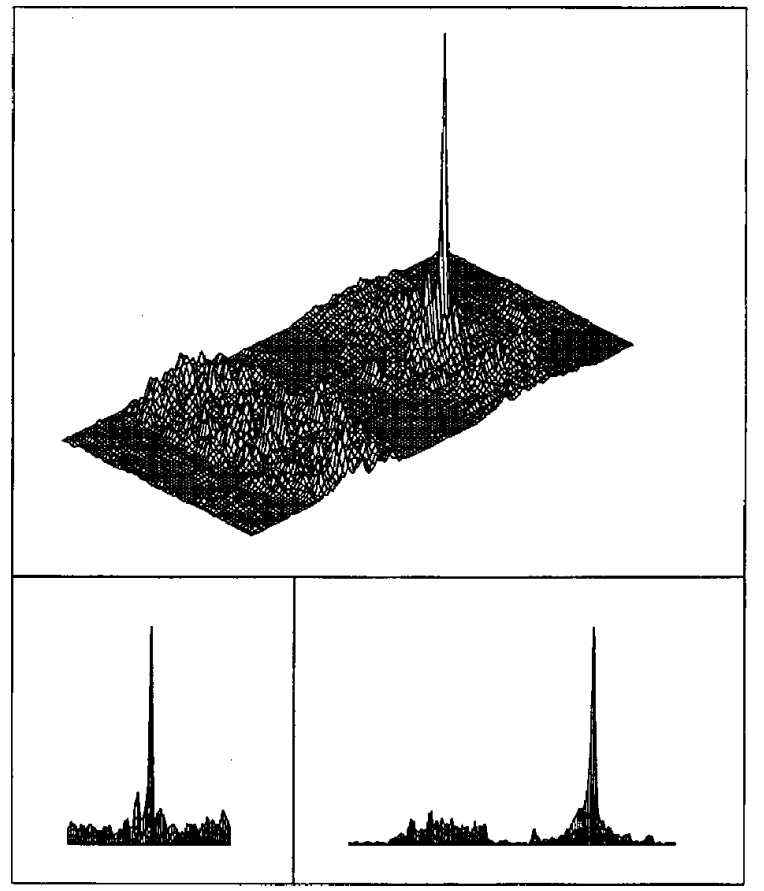

Fig. 15. Correlation between the binary-phase-only SDF and the input scene of Fig. 13.

ity between the SDF and the constrained filter. The change of the similarity criterion might permit us to obtain a solution when this is not feasible with the Euclidean distance. This possibility is also being studied, and the results will be reported in a future work.

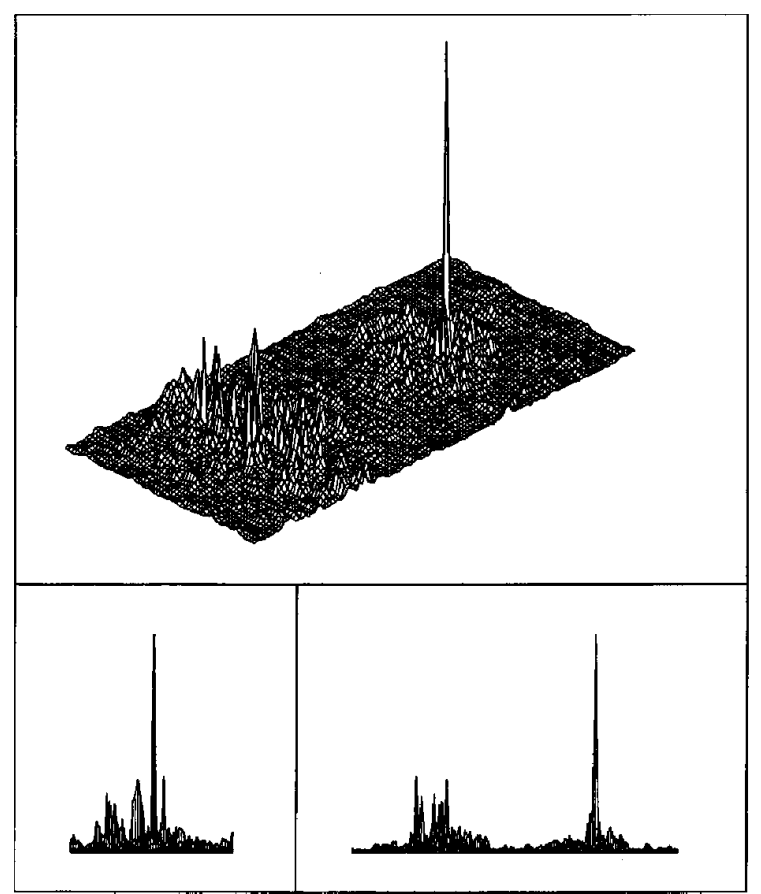

Fig. 16. Correlation between the spirally constrained SDF and the input scene of Fig. 13.

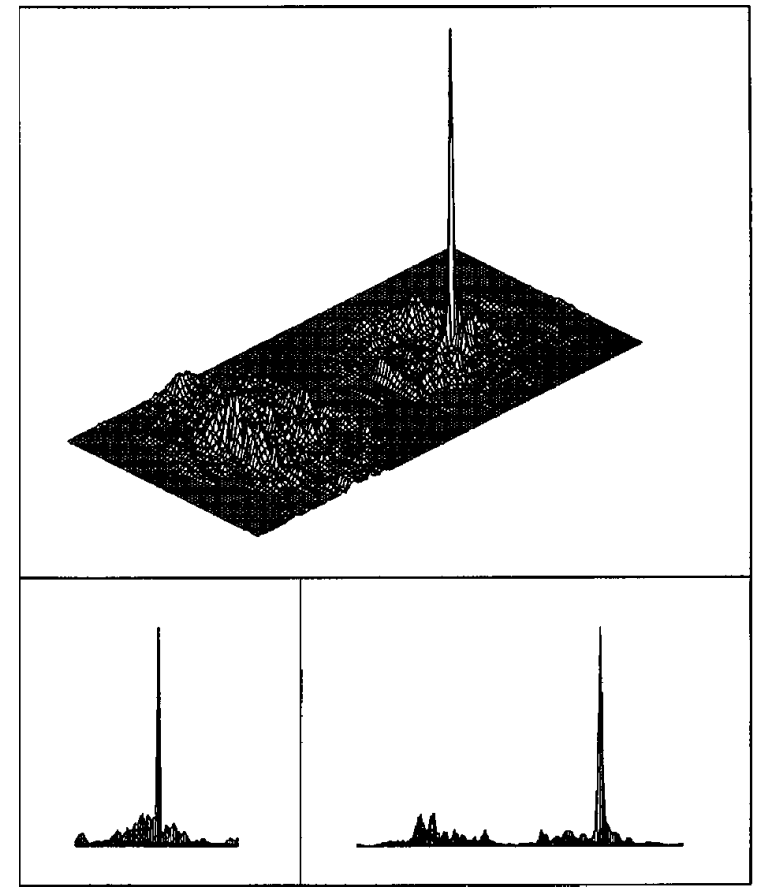

Fig. 17. Correlation between the arbitrarily constrained SDF [Fig. 6(d)] and the input scene of Fig. 13.

\section{Appendix A}

We derive an expression for the scaling of the central correlations leading to minimum error, as mentioned in Section 4.

The expression for the error function to be minimized can be written in vector notation as [see Eq. (10)]

$$
E=\mathbf{h}^{+} \mathbf{h}-\mathbf{a}^{+} \mathbf{h}-\mathbf{h}^{+} \mathbf{a}+\mathbf{a}^{+} \mathbf{a},
$$

where $\mathbf{h}$ is the MED-SDF filter:

$$
\mathbf{h}=\alpha \mathbf{c o m p}+\mathbf{P a}
$$

and $\alpha$ is a real constant that scales the central correlations. By substituting Eq. (A.2) into Eq. (A.1) we get

$$
\begin{aligned}
E(\alpha)= & \left.\alpha(\operatorname{comp})^{+}+\mathbf{a}^{+} \mathbf{P}\right](\alpha \operatorname{comp}+\mathbf{P a}) \\
& -\mathbf{a}^{+}(\alpha \mathbf{c o m p}+\mathbf{P a}) \\
& -\left[\alpha(\operatorname{comp})^{+}+\mathbf{a}^{+} \mathbf{P}\right] \mathbf{a}+\mathbf{a}^{+} \mathbf{a} \\
= & \alpha^{2}(\operatorname{comp})^{+} \mathbf{c o m p}+\alpha(\operatorname{comp})^{+} \mathbf{P a} \\
& +\alpha \mathbf{a}^{+} \mathbf{P}(\operatorname{comp})+\mathbf{a}^{+} \mathbf{P}^{2} \mathbf{a}-\alpha \mathbf{a}^{+} \operatorname{comp}-\mathbf{a}^{+} \mathbf{P a} \\
& -\alpha(\operatorname{comp})^{+} \mathbf{a}-\mathbf{a}^{+} \mathbf{P a}+\mathbf{a}^{+} \mathbf{a} .
\end{aligned}
$$

Owing to the properties of the projection operator $\mathbf{P}$,

$(\mathbf{c o m p})^{+} \mathbf{P a}=0$,

$\mathbf{a}^{+} \mathbf{P}(\mathbf{c o m p})=0$,

$\mathbf{a}^{+} \mathbf{P}^{2} \mathbf{a}=\mathbf{a}^{+} \mathbf{P a} \Rightarrow \mathbf{a}^{+} \mathbf{P}^{2} \mathbf{a}-\mathbf{a}^{+} \mathbf{P a}=0$, 
we can write

$$
E(\alpha)=\alpha^{2}(\operatorname{comp})^{+} \operatorname{comp}-2 \alpha \mathbf{a}^{+} \operatorname{comp}-\mathbf{a}^{+} \mathbf{P a}+\mathbf{a}^{+} \mathbf{a},
$$

and, finally, by setting the derivative of $E$ to 0 , we find the expression for $\alpha$ leading to minimum error:

$$
\frac{\partial E}{\partial \alpha}=0 \Leftrightarrow 2 \alpha(\operatorname{comp})^{+} \operatorname{comp}-2 \mathbf{a}^{+} \operatorname{comp}=0,
$$

whence

$$
\alpha=\frac{\mathbf{a}^{+} \operatorname{comp}}{(\operatorname{comp})^{+} \operatorname{comp}}
$$

This work has been partially supported by the Spanish Comisión Interministerial de Ciencia y Tecnología under grants TAP94-0303 and TAP93-0667C03-01.

\section{References}

1. B. V. K. Vijaya Kumar, "Tutorial survey of composite filter designs for optical correlators," Appl. Opt. 31, 4773-4801 (1992).

2. A. Mahalanobis, B. V. K. Vijaya Kumar, and D. Casasent, "Minimum average correlation energy filters," Appl. Opt. 26, 3633-3640 (1987).

3. B. V. K. Vijaya Kumar, "Minimum variance synthetic discriminant functions," J. Opt. Soc. Am. A 3, 1579-1584 (1986).

4. Ph. Réfrégier, "Optimal trade-off filters for noise robustness, sharpness of the correlation peak and Horner efficiency," Opt. Lett. 16, 829-831 (1991).

5. Ph. Réfrégier, "Méthodes de reconnaissance des formes pour la corrélation optique," Rev. Tech. Thomson-CSF 22, 649-734 (1990).

6. R. D. Juday, "Optimal realizable filters and the minimum Euclidean distance principle," Appl. Opt. 32, 5100-5111 (1993).

7. V. Laude and Ph. Réfrégier, "Multicriteria characterization of coding domains with optimal Fourier spatial light modulators filters," Appl. Opt. 33, 4465-4471 (1994).
8. J. L. Horner and P. D. Gianino, "Applying the phase-only filter concept to the synthetic discriminant function correlation filter," Appl. Opt. 24, 851-855 (1985).

9. D. Casasent and W. A. Rozzi, "Computer-generated and phaseonly synthetic discriminant function filters," Appl. Opt. 25, 3767-3772 (1986).

10. R. R. Kallman, "Optimal low noise phase-only and binary phase-only optical correlation filters for threshold detectors," Appl. Opt. 25, 4216-4217 (1986).

11. D. A. Jared and D. J. Ennis, "Inclusion of filter modulation in synthetic-discriminant-function construction," Appl. Opt. 28, 232-239 (1989).

12. Z. Bahri and B. V. K. Vijaya Kumar, "Algorithms for designing phase-only synthetic discriminant functions," in Optical Information Processing Systems and Architectures, B. Javidi, ed., Proc. Soc. Photo-Opt. Instrum. Eng. 1151, 138-147 (1989).

13. D. W. Carlson and B. V. K. Vijaya Kumar, "Synthetic discriminant functions for implementation on arbitrarily constrained devices," in Optical Information Processing Systems and Architectures IV, B. Javidi, ed., Proc. Soc. Photo-Opt. Instrum. Eng. 1772, 10-20 (1992).

14. U. Mahlab and J. Shamir, "Phase-only entropy-optimized filter generated by simulated annealing," Opt. Lett. 14, 1168-1170 (1989).

15. J. Rosen and J. Shamir, "Application of the projection-ontoconstraint-sets algorithm for optical pattern recognition," Opt. Lett. 16, 752-754 (1991).

16. D. C. Youla and H. Webb, "Image restoration by the method of convex projections: Part 1-theory," IEEE Trans. Med. Imaging 1, 81-94 (1982).

17. Z. Bahri and B. V. K. Vijaya Kumar, "Generalized synthetic discriminant functions,” J. Opt. Soc. Am. A 5, 562-571 (1988).

18. B. V. K. Vijaya Kumar, Z. Bahri, and A. Mahalanobis, "Constrained phase optimization in minimum variance synthetic discriminant functions," Appl. Opt. 27, 409-413 (1988).

19. Ph. Réfrégier and J. P. Huignard, "Phase selection of synthetic discriminant functions filters," Appl. Opt. 29, 4772-4778 (1990).

20. B. V. K. Vijaya Kumar and L. Hassebrook, "Performance measures for correlation filters," Appl. Opt. 29, 2997-3006 (1990). 